\title{
The Shape of The Exfoliated Surface during Femtosecond Laser Ablation
}

\author{
Naoya Kakimoto, Takashi Eyama, Rui Izutsu, and Takuro Tomita \\ Faculty of Engineering, Tokushima University, \\ Tokushima 770-8506, Japan
}

E-mail: tomita@tokushima-u.ac.jp

\begin{abstract}
Femtosecond laser ablation processes on metals are systematically observed using a Ti:sapphire laser pump and the soft x-ray laser probe reflective imaging system. In addition to the dark disk at the femtosecond laser-irradiated spot, an elliptical shadow that expands in the horizontal direction in the nanosecond time domain was observed. We show that the shadow is caused by the exfoliated surface during femtosecond laser ablation. By analyzing the shadow, we can determine the evolution of the exfoliated surface over time. The shape of the film is the result of the relationship between the spatial distribution of the laser beam profile and the ablation threshold of the metal.
\end{abstract}

DOI: $10.2961 /$ jlmn.2016.01.0017

Keywords: femtosecond laser ablation, time-resolved measurement, soft x-ray laser probe, reflective imaging, metals

\section{Introduction}

Recently, the phenomena caused by femtosecond laser ablation have been studied extensively. Femtosecond laser processing, such as cutting of materials, has attracted much interest because of its potential application to the microfabrication of transparent materials. Many interesting phenomena related to multiple pulses of femtosecond laser irradiation have been reported [1-12]. Two examples are the femtosecond laser-induced ripples that appear on a solid surface that are its subwavelength periodic structure [13, $14]$ and the ablation rate per one laser shot that is far lower than $1 \mathrm{~nm} /$ pulse [15]. These phenomena are observed only with femtosecond laser irradiation, not nanosecond or continuous wave laser irradiation. They may lead to novel materials processing techniques because of their potential for precise materials processing. However, the mechanism of femtosecond laser ablation has not been investigated.

Earlier studies on femtosecond laser ablation found that immediately ( $\sim 20 \mathrm{ps}$ ) after femtosecond laser irradiation, dark disks emerged at the center of the laser-irradiated area [16]. Simultaneously, dilatation of the sample surface was observed for up to 50 ps [17]. Regardless of the slight difference in time, these phenomena are very similar for the three metals that we used in our experiment: $\mathrm{Au}, \mathrm{W}$, and Pt.

The soft x-ray laser probe has several advantages over the visible light probe. One is that laser-induced plasma is transparent to soft x-rays, which enables the acquisition of clear images of the laser-irradiated surface during ablation. Second, the soft x-ray laser probe has the potential for greater spatial resolution than the visible light probe. Thus, for our study we employed the infrared laser pump and the soft x-ray laser probe system.

Time-resolved single-shot reflective imaging successfully observed the initial process of femtosecond laser ablation. In our previous study [16-21], we observed Newton's rings $100-1000$ ps after femtosecond laser irradiation. The rings are due to interference of the soft x-ray beam that is either reflected or transmitted by the exfoliated thin film resulting from ablation. The appearance of Newton's rings shows that the exfoliated thin film is smooth enough to reflect soft x-rays at its boundaries. The development of Newton's rings over time provides detailed information on the dilatation of the exfoliated thin film. In addition, we successfully determined the shape of the exfoliated surface in the nanosecond time domain using a soft x-ray shadowgraph of the expanded dome. In this paper, we report on femtosecond laser ablation of $\mathrm{Au}, \mathrm{W}$, and $\mathrm{Pt}$ surfaces in the nanosecond time domain.

\section{Experimental}

The soft X-ray laser system, constructed by the Japan Atomic Energy Agency (JAEA), was used as the probe beam. The system provides 7 ps soft x-ray pulses and delivers $>10^{9}$ soft x-ray photons of $89.2 \mathrm{eV}(13.9 \mathrm{~nm})$. The brighter laser pulses make it possible to capture ultrafast phenomena in a single x-ray pulse. Figure 1(a) is a schematic of the femtosecond laser pump and the soft x-ray laser probe system. Measurements were performed in a vacuum at a pressure $<10^{-3} \mathrm{~Pa}$. A focusing mirror focused the soft x-ray in front of the sample, whereon it was defocused by two injection mirrors onto the sample surface. The incident angle of the soft x-ray on the sample surface was about $20^{\circ}$. The reflectivity of soft $\mathrm{x}$-ray on $\mathrm{Au}$ and $\mathrm{Pt}$ at this angle is about $40 \%$ and that on $\mathrm{W}$ is about $30 \%$ [22]. An imaging mirror then transferred the sample image onto the detector. The magnification factors by the imaging mirror were about 19.1 for $\mathrm{Au}$ and about 38.2 for $\mathrm{W}$ and Pt. A change in the experimental setup for each sample caused the difference in magnification. The detector was a back illuminated charge-coupled device (CCD) for soft x-rays (Princeton Instruments, PIXIS-XO: 2048B).

The pump laser was a Ti:sapphire laser with a Gaussian lateral intensity profile. The duration of a laser pulse was $80 \mathrm{fs}$ and the central wavelength of the laser beam was 

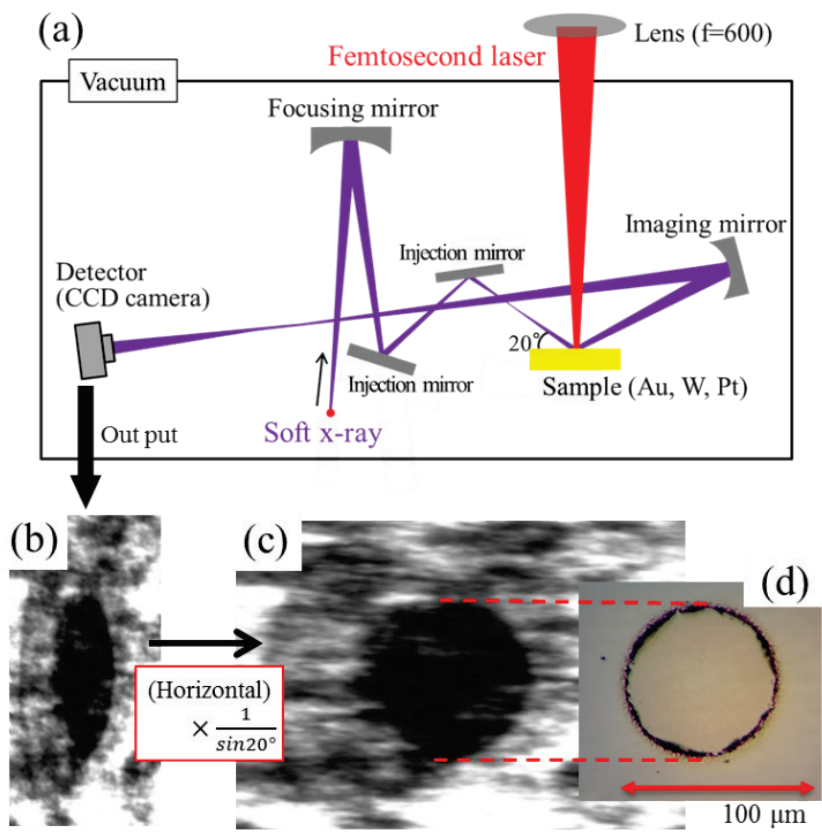

Figure 1. (a) Schematic of the femtosecond laser pump and the soft $\mathrm{x}$-ray laser probe system. (b) Output image from the detector after femtosecond laser irradiation of $\mathrm{Au}$. (c) Image in (b) expanded horizontally $1 / \sin 20^{\circ}$ times while the vertical remained the same. (d) Optical microscopy image of ablation crater.

$795 \mathrm{~nm}$. The emitted pulse was focused by a lens $(f=600 \mathrm{~mm})$ onto the sample surface at nearly normal incidence. Focal spot sizes (full width at half maximum, FWHM) of the pump laser beam on the sample surface were about $100 \mu \mathrm{m}$. The pumping energy was adjusted by a combination of a half-wavelength plate and a polarizing beam splitter. To evaluate the pulse energy of each shot on the sample, a photodiode joulemeter (OPHIR, PD10-SHV2) sampled a fraction of the incident pulse.

Each sample was evaporated onto a fused silica substrate. The $\mathrm{Au}, \mathrm{W}$, and Pt layers were 100, 113, and $100 \mathrm{~nm}$ thick, respectively. These metals were chosen as samples because of their high reflectivity to soft x-rays and their wide range of melting points. These characteristics enabled us to observe the femtosecond laser ablation dynamics more easily and thus enable discussion of the effect of a material's properties on the ablation phenomena. An atomic force microscope (AFM) measured the initial surface roughness; the root mean square (rms) deviations of the roughness of all sample surfaces were $<1.0 \mathrm{~nm}$.

\section{Results and Discussion}

Soft x-ray reflective imaging of $\mathrm{Au}, \mathrm{W}$, and Pt was performed using the setup shown Fig. 1(a). Soft X-ray reflectivity is quite sensitive to surface roughness. If the rms surface roughness of $\mathrm{Au}$ is $5 \mathrm{~nm}$, soft x-ray reflectivity decreases to $15 \%$. The output image of the detector after femtosecond laser irradiation of $\mathrm{Au}$ is shown in Fig. 1(b). For our study, we used images that were expanded along the horizontal by $1 / \sin 20^{\circ}$ times, such as that shown in Fig. 1(c). That image is the same as the optical microscopy image in Fig. 1(d). These images show that the reflectivity of soft x-rays on the femtosecond laser-irradiated area, which is often roughened by the irradiation, is significantly reduced, but it is not reduced outside of the laser-irradiated area. However, in addition to the dark disk at the center of the irradiated spot, an elliptical shadow was observed in the horizontally expanded image, outside of the femtosecond laser-irradiated area. This shadow, a typical example of which is shown in Fig. 2(a), can be observed from 20 to $500 \mathrm{~ns}$ after femtosecond laser irradiation. The mechanism of the origin of this shadow is shown Fig. 2(b). The numbered optical paths correspond to the areas in the soft x-ray reflective image [Fig. 2(a)]. Optical path (1) represents the soft x-ray that reflects off the ablated crater and passes the exfoliated thin film twice, i.e., incoming and outgoing, on its way to the dome formed by the exfoliated thin film. Thus, the soft x-ray photon count from path (1) on the CCD is the lowest. Optical path (2) shows that the soft x-ray passes the exfoliated thin film, goes into the dome, and passes the exfoliated thin film again. However, the soft $x-$ ray is not reflected on the ablated crater, which would cause a drastic decrease in soft x-ray reflectivity because of its rough surface. Optical path (3) indicates that the soft $\mathrm{x}$ ray did not reflect off the ablated crater or pass the exfoliated thin film. The area marked (3) in the CCD image shows that the soft $\mathrm{x}$-ray reflectivity was not affected by the flat surface, i.e., the nonirradiated area. Thus, the CCD image can be divided into three areas: the round dark disk at the center, the elliptical shadow outside the dark disk, and the area with no significant change. These results suggest that the elliptical shadow evolves over time because of the expansion of the exfoliated thin film after femtosecond laser irradiation. Because the configuration for soft x-ray irradiation was fixed for all measurements, e.g., the incident angle of the soft $x$-ray was about $20^{\circ}$. It is not difficult to estimate the vertical spatial profiles of the exfoliated thin films.

Figure 3(a) shows the change in the soft x-ray reflectivity on $\mathrm{Au}$ over time. The peak fluence of the femtosecond

(a)

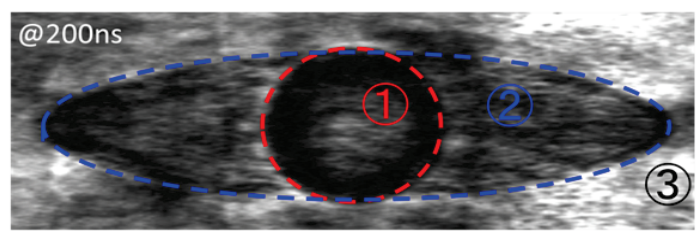

(b)

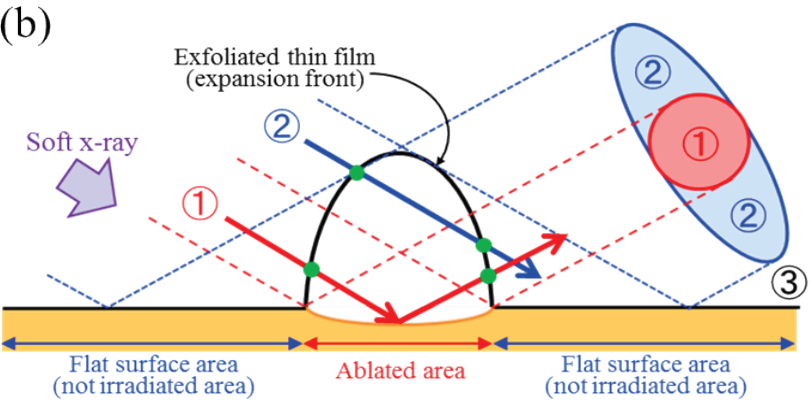

Figure 2. (a) Typical shadowgraph of $\mathrm{Au} 200 \mathrm{~ns}$ after femtosecond laser irradiation. (b) Mechanism of the origin of the shadow. (1) corresponds to the area in the red circle in (a), (2) corresponds to the area in the blue ellipse in (a), and (3) corresponds the area outside of the blue ellipse in (a). 
laser pulse was $1.6 \mathrm{~J} / \mathrm{cm}^{2}$. The elliptical shadow extended in the horizontal direction over time. Figure 3(b) shows the shapes of the exfoliated surface on Au estimated using the elliptical shadow on the shadowgraph at each delay time. The shapes of the elliptical shadows were fitted by a Gaussian curve, indicating that the shape of the exfoliated surface also had a Gaussian vertical profile.

Figures 4(a) and (b) show the soft x-ray shadowgraph and the evolution of the shape of the exfoliated surface on W over time. Figures 4(c) and 4(d) show the soft x-ray shadowgraph and the evolution of the shape of the exfoliated surface on Pt over time. Peak fluence was $1.0 \mathrm{~J} / \mathrm{cm}^{2}$ for $\mathrm{W}$ and $0.7 \mathrm{~J} / \mathrm{cm}^{2}$ for Pt. Similar to that of $\mathrm{Au}$, the elliptical shadows of $\mathrm{W}$ and $\mathrm{Pt}$ extended in the horizontal direction and their shapes over time were well fitted by a Gaussian curve.

The shape of the exfoliated surface over time is fitted
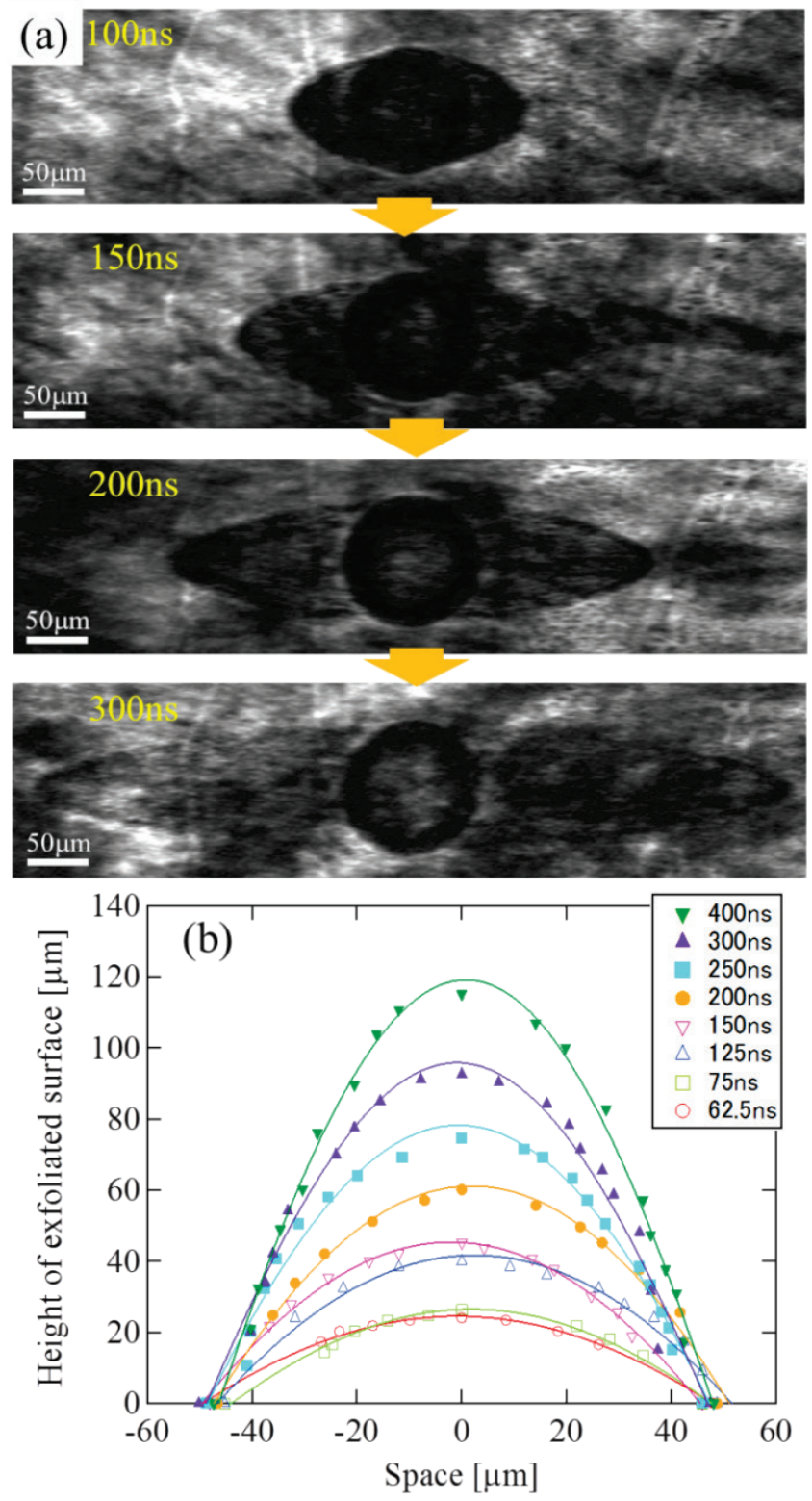

Figure 3. (a) Soft $x$-ray shadowgraphs showing the evolution of soft x-ray reflectivity on Au over time. (b) Shape of the exfoliated surface on Au estimated from the soft x-ray shadowgraph at each delay time. The markers indicate measured points fitted by the solid lines. by a Gaussian curve because the radial distribution of the intensity of the femtosecond laser has a Gaussian-shaped profile. If we assume that the input energy of the laser was linearly converted into kinetic energy in the exfoliated thin film, the spatial velocity distribution and thus the height of the exfoliated thin film should have Gaussian-shaped profiles.

Comparing Fig. 3(b), 4(b) and 4(d), the tail of the exfoliated film are different for the three metals. This difference can be explained by the relationship between the ablation threshold of the metal and the intensity profile of the irradiating laser beam. Some laser energy is used to break the bonds between atoms in the metals and the remainder is converted into the kinetic energy of the fragmented atoms of the exfoliated surfaces (Fig. 5). This fluence relationship is expressed by
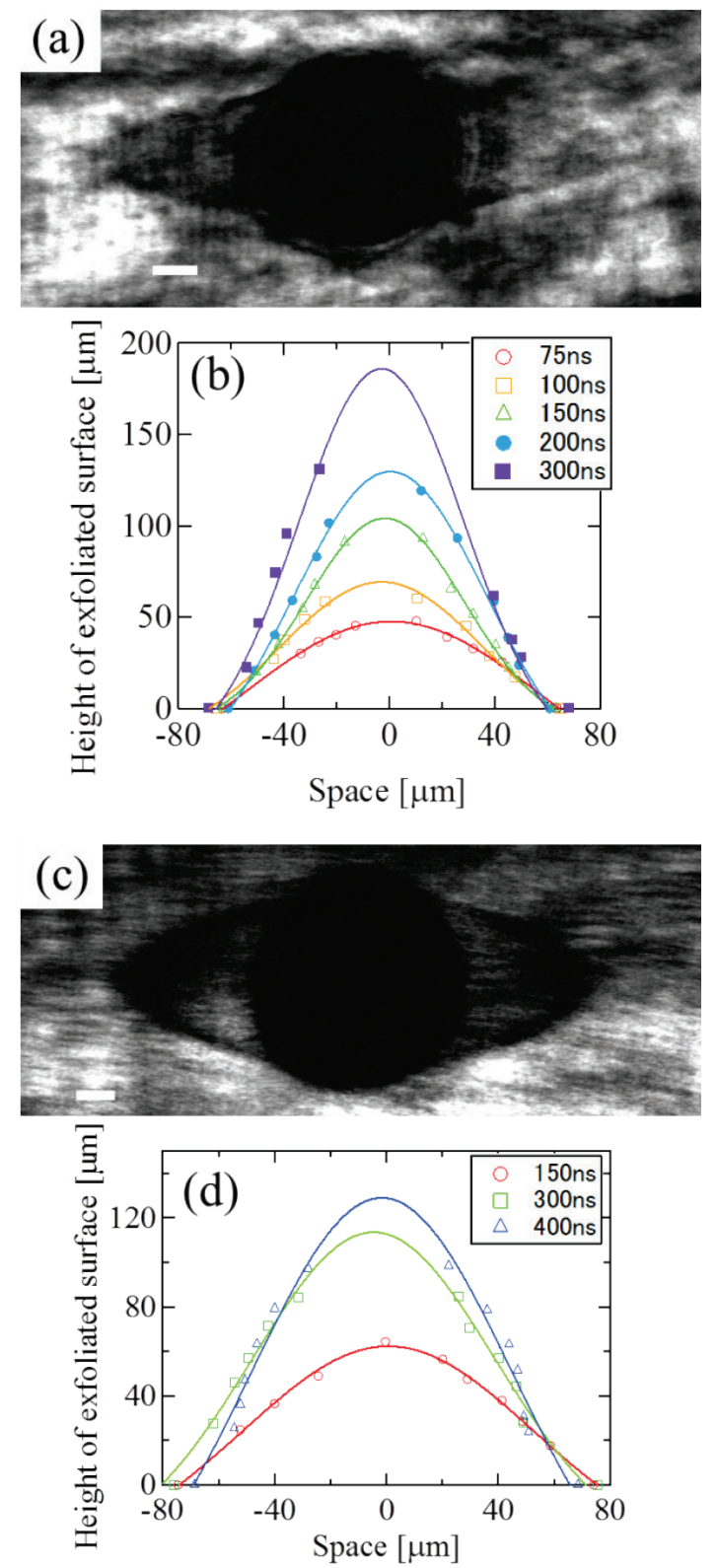

Figure 4. (a) Soft x-ray shadowgraph on W. (b) Shapes of the exfoliated surface on $\mathrm{W}$ at each delay time. (c) Soft xray shadowgraph on Pt. (d) Shapes of the exfoliated surface on $\mathrm{Pt}$ at each delay time. The markers indicate measuring points fitted by the solid lines. The scale bar $=50 \mu \mathrm{m}$ in (a) and (c). 


$$
F_{\text {kinetic energy }}=F_{\text {laser energy }}-F_{\text {ablation threshold }}
$$

The fluences of the ablation thresholds of $\mathrm{Au}, \mathrm{W}$, and $\mathrm{Pt}$ are approximately $0.8,0.4$, and $0.2 \mathrm{~J} / \mathrm{cm}^{2}$, respectively. Thus, the shapes of the exfoliated surfaces of the three metals are different but they are all fitted by a Gaussian curve.

The findings of this study provide insight into the initial process of femtosecond laser ablation.
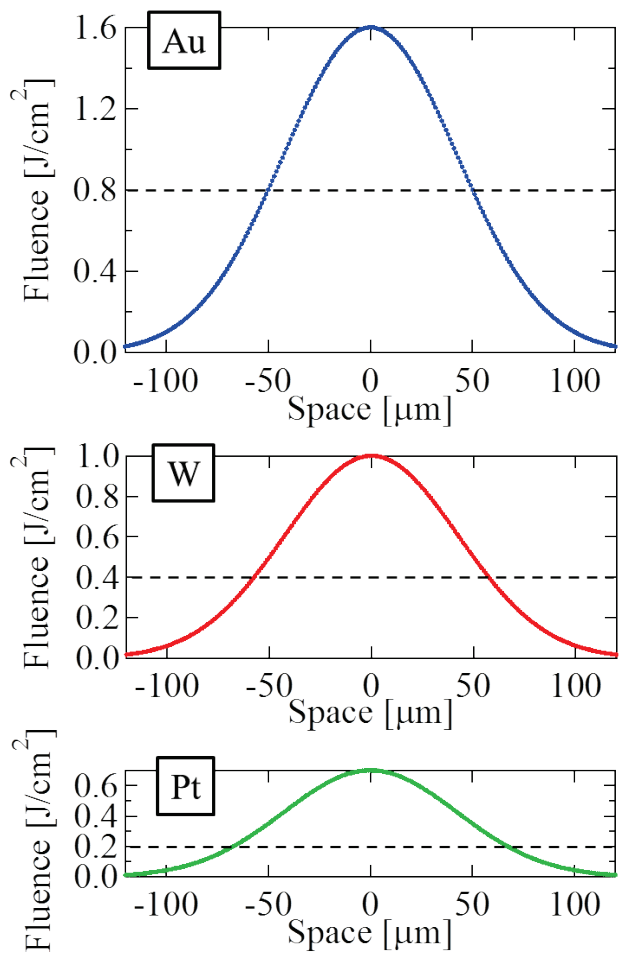

Figure 5. The solid lines are the radial distributions of the intensity of the femtosecond laser and the dotted lines are the ablation thresholds of the metal.

\section{Conclusion}

A Ti:sapphire laser pump and the soft $\mathrm{x}$-ray laser probe reflective imaging system were used to observe femtosecond laser ablation dynamics on $\mathrm{Au}, \mathrm{W}$, and $\mathrm{Pt}$. We found that the laser irradiation exfoliated the surface of the sample. The exfoliated thin films were observed as horizontally expanded elliptical shadows in the oblique incident soft $\mathrm{x}$ ray shadowgraphs. The shadows were observed tens to hundreds of nanoseconds after femtosecond laser irradiation. Similar to the intensity profile of femtosecond laser irradiation, the shape of the exfoliated surface was Gaussian. These results suggest that the shape of the exfoliated surface can be controlled by controlling the radial profile of the intensity of the femtosecond laser beam.

\section{Acknowledgments}

This study was performed with the help of the Facilities Utilization System of the Japan Atomic Energy Agency. We thank Prof. Tohru Suemoto, Dr. Yasuo Minami, and the JAEA staff for technical assistance and valuable discussions. This work was supported in part by a JSPS
KAKENHI Grant-in-Aid for Scientific Research (B) (grant No. 25286086).

\section{References}

[1] R. L. Fork, C. H. Brito Cruz, P. C. Becker and C. V. Shank, Opt. Lett. 12, (1987) 483.

[2] E. J. Takahashi, P. Lan, O. D. Mücke, Y. Nabekawa and K. Midorikawa, Nat. Commun. 4, (2013) 2691.

[3] S. Kawata, H. B. Sun, T. Tanaka and K. Takada, Nature 412, (2001) 697.

[4] A. Marcinkevičius, S. Juodkazis, M. Watanabe, M. Miwa, S. Matsuo, H. Misawa and J. Nishii, Opt. Lett. 26, (2001) 277.

[5] Y. Hayasaki, M. Isaka, A. Takita and S. Juodkazis, Opt. Express 19, (2011) 5725.

[6] D. Perez and L. J. Lewis, Phys. Rev. B 67, (2003) 184102.

[7] P. Lorazo, L. J. Lewis and M. Meunier, Phys. Rev. B 73, (2006) 134108.

[8] L. V. Zhigilei, Z. Lin and D.S. Ivanov, J. Phys. Chem. C 113, (2009) 11892

[9] M. Yamaguchi, S. Ueno, R. Kumai, K. Kinoshita, T. Murai, T. Tomita, S. Matsuo and S. Hashimoto, Appl. Phys. A 99, (2010) 23.

[10] Y. Izawa, Y. Setuhara, M. Hashida, M. Fujita and Y. Izawa, Jpn. J. Appl. Phys. 45, (2006) 5791.

[11] M. Deki, T. Ito, M. Yamamoto, T. Tomita, S. Matsuo, S. Hashimoto, T. Kitada, T. Isu, S. Onoda and T. Ohsima, Appl. Phys. Lett. 98, (2011) 133104.

[12] B. N. Chichkov, C. Momma, S. Nolte, F. von Alvensleben and A. Tünnermann Appl. Phys. A 63, (1996) 109115.

[13] T. Tomita, K. Kinoshita, S. Matsuo and S. Hashimoto, Appl. Phys. Lett. 90, (2007) 153115.

[14] T. Tomita, K. Kinoshita, S. Matsuo and S. Hashimoto, Jpn. J. Appl. Phys. 45, (2006) L444.

[15] M. Hashida, A. F. Semerok, O. Gobert, G. Petite and J. F. Wagner, Proc. SPIE 4423, (2001) 178.

[16] T. Tomita, M. Nishikino, N. Hasegawa, Y. Minami, R. Takei, M. Baba, T. Eyama, S. Takayoshi, T. Kaihori, T. Morita, Y. Hirano, T. Kawachi, M. Yamagiwa and T. Suemoto, J. Laser Micro/Nanoeng. 9, (2014) 137.

[17] M. Nishikino, N. Hasegawa, T. Tomita, Y. Minami, R. Takei, M. Baba, T. Eyama, S. Takayoshi, T. Kawachi, M. Yamagiwa and T. Suemoto, SPIE Opt. 8849, (2013).

[18] M. Baba, M. Nishikino, N. Hasegawa, T. Tomita, Y. Minami, R. Takei, M. Yamagiwa, T. Kawachi and T. Suemoto, Jpn. J. Appl. Phys. 53, (2014) 080302.

[19] T. Tomita, M. Yamamoto, N. Hasegawa, K. Terakawa Y. Minami, M. Nishikino, M. Ishino, T. Kaihori, Y. Ochi, T. Kawachi, M. Yamagiwa and T. Suemoto, Opt. Express 20, (2012) 29329.

[20] Y. Ochi, K. Terakawa, N. Hasegawa, M. Yamamoto, T. Tomita, T. Kawachi, Y. Minami, M. Nishikino, T. Imazono, M. Ishino and T. Suemoto, Jpn. J. Appl. Phys. 51, (2012) 016601.

[21] T. Suemoto, K. Terakawa, Y. Ochi, T. Tomita, M Yamamoto, N. Hasegawa, M. Deki, Y. Minami and T. Kawachi, Opt. Express 18, (2010) 14114.

[22] X-Ray Interactions With Matter: http://henke.lbl.gov/optical_constants/

(Received: May 22, 2015, Accepted: January 26, 2016) 